\title{
Aprendizajes en relación con la enseñanza en carreras de formación inicial, qué se aprende y cómo
}

Datos de contacto:

Maria alejandra Salgueiro Universidad Nacional de Córdoba

asalguei@live.com.ar

Recibido: 09/11/2020 Aceptado: 01/02/2021
María Alejandra SALGUEIRO

\section{RESUMEN}

La formación de profesores es un campo muy explorado en la investigación, no obstante, se observa cierta vacancia en el estudio sobre algunos aspectos, especialmente en relación con los aprendizajes alcanzados en las carreras de formación inicial. El presente artículo expone una síntesis de los resultados de una tesis doctoral llevada a cabo en la Universidad de Málaga, España, cuyo objetivo fue indagar acerca de qué aprenden quienes egresan de una carrera de formación docente inicial acerca de la enseñanza y cómo lo logran. Indagación que se llevó a cabo en el contexto de un profesorado destinado a graduados universitarios que se desarrolla en la Universidad Nacional de Córdoba, Argentina. Este fenómeno se abordó desde una metodología de tipo cualitativa, se consideró principalmente el punto de vista de los sujetos involucrados: egresados y profesores de la carrera. Se priorizaron como técnicas de relevamiento la entrevista y los grupos focales.

Los resultados alcanzados permiten conceptualizar a los aprendizajes como articulación entre tipos de aprendizaje, modalidades y condiciones. Se identifican aprendizajes cuyo componente fundamental es de orden cognitivo y sobre todo aprendizajes que remiten a experiencias y vivencias. Se destaca el valor que tienen para los egresados estos últimos. Se advierte sobre la especificidad que cobran los aprendizajes logrados en el marco de este tipo particular de formación, caracterizados como pasajes.

Se concluye acerca de la necesidad de que las propuestas de enseñanza de los profesorados presenten más atención sobre lo aprendizajes valorados por los egresados, así como las maneras de alcanzarlos.

PALABRAS CLAVE: formación docente inicial; aprendizajes; saberes; enseñanza 


\title{
Learning in relation to teaching in initial training careers, what is learned and how
}

\begin{abstract}
Although the training of teachers has been the object of investigation, there is a vacancy in some of the aspects that compromises this problem, especially with respect to the learning achieved in initial training careers. This article presents a synthesis of the results of a doctoral thesis carried out at the University of Malaga, Spain, the objective of which was to inquire about what those who graduate from an initial teacher training career learn about teaching and how they achieve it. An inquiry that was carried out in the context of a teaching staff for university graduates that takes place at the National University of Córdoba, Argentina. This phenomenon was approached from a qualitative methodology, the point of view of the subjects involved was mainly considered: graduates and professors of the career. The interview and focus groups were prioritized as survey techniques.

The results achieved allow the conceptualization of learning as an articulation between types of learning, modalities and conditions. Learning whose fundamental component is cognitive and above all learning that refers to experiences are identified. The value of the latter for graduates is highlighted. It warns about the specificity of the learning achieved in the framework of this particular type of training, characterized as passages.

It is concluded about the need for the teaching proposals of the teachers to present more attention on the learning valued by the graduates, as well as the ways to achieve them.
\end{abstract}

KEYWORDS: initial teacher training; learning; knowledge; teaching

\section{Introducción}

Si bien la formación de profesores ha sido objeto de estudio en diversas investigaciones tanto en Argentina como a nivel internacional se observa vacancia en algunos de los aspectos que compromete esta problemática. Una cuestión que no ha sido muy explorada remite qué aprenden los egresados de las carreras de formación inicial en particular, en relación con la tarea sustantiva del profesor, es decir, la enseñanza.

Tatiana Cisternas (2011) describe los territorios explorados y no explorados de la formación de profesores, evidencia cierta limitación en la investigación sobre este campo, observa una tendencia a estudiar ciertos focos problemáticos sobre otros y da cuenta de la invisibilidad o ausencia de algunos aspectos de la formación que no parecen ser materia de estudio. Reconoce que uno de estos aspectos precisamente 
son los aprendizajes alcanzados por quienes egresan de una carrera de profesorado: Los aprendizajes, las prácticas, las dificultades del egresado de pedagogía, nos parece una de las omisiones más preocupantes y de mayores consecuencias dentro del conjunto de objetos invisibilizados por la investigación nacional en este campo. El estudiante que recibe una formación inicial y que luego de cumplir su período formativo egresa con un título que lo habilita para desempeñar su profesión resulta ser un actor marginal si se lo compara con los otros actores investigados y dentro del conjunto total de investigaciones (Cisternas, 2011, p. 16).

En relación a las carreras de profesorado de carácter consecutivo destinadas a graduados universitarios, los hallazgos son aún más escasos. Los trabajos y aportes sobre esta cuestión no remiten necesariamente a estudios de carácter investigativo. Se trata de trabajos que intentan describir y problematizar cuestiones vinculadas a los modelos de formación propuestos en estas carreras, a las maneras en que se resuelve la formación pedagógica y su relación con la formación de base de quienes aspiran a desempeñarse como docentes, a los dispositivos de prácticas profesionalizantes. Asimismo, la problemática es analizada generalmente desde la perspectiva de la enseñanza y de los profesores.

El presente artículo expone una síntesis de los resultados de una tesis doctoral llevada a cabo en la Universidad de Málaga, España, cuyo objetivo fue analizar qué aprenden quienes egresan de una carrera de profesorado en relación con la enseñanza y cómo lo logran. Indagación que se llevó a cabo en el contexto de una carrera de formación destinada a graduados universitarios que se desarrolla en la Universidad Nacional de Córdoba, Argentina. Este tipo de carrera otorga cierta particularidad al estudio pues se trata de aprender otra práctica profesional diferente de la que prepara la formación de base.

Este fenómeno se abordó desde una metodología de tipo cualitativa que toma en consideración el punto de vista de los sujetos involucrados: egresados y profesores de la carrera. Se priorizaron como técnicas de relevamiento la entrevista y los grupos focales. Respecto de los egresados se focalizó en la experiencia de formación vivida en orden a identificar aquello que ellos consideran haber aprendido en relación con la enseñanza y de qué modo. Respecto de los profesores se consideraron no sólo sus propuestas de enseñanza sino también sus percepciones sobre los aprendizajes de los estudiantes de la carrera.

Los datos relevados y su análisis permiten conceptualizar a los aprendizajes como resultado de la articulación entre tipos de aprendizaje, modalidades y condiciones.

Respecto del tipo de aprendizajes se identifican aquellos cuyo componente fundamental es de orden cognitivo y también aprendizajes que remiten a experiencias y vivencias. Se destaca el valor que tienen para los egresados estos últimos.

Se advierte sobre la especificidad que cobran los aprendizajes logrados en el marco de este tipo particular de formación, caracterizados como "pasajes". Pasajes que a su vez tienen cierta particularidad porque suponen no solo pasar de ser estudiante a ser profesor, sino que también implicó pasar del campo de conocimiento 
de la especialidad de los cursantes al campo pedagógico y didáctico.

Es recurrente el aprender a estar en la clase. Para los egresados significó apropiarse de ese espacio, pararse, situarse en ese lugar y ponerse de "ese otro lado", fue la experiencia más significativa porque a través de ella pudieron dar "el paso", ingresar al territorio escolar y participar con cierta intensidad de "comunidades de práctica".

Los resultados coinciden con el diagnóstico extendido sobre los programas de formación de profesores: la necesidad de prestar mayor atención a los aprendizajes valorados por quienes cursan estas carreras los cuales remiten a las demandas reales de la profesión. Muestran que la formación docente inicial, si bien es una temática que es objeto de debate en los ámbitos académicos y políticos y en consecuencia provocado cambios sustanciales en los currículos, es una problemática no del todo resuelta en las propuestas de enseñanza y en los proyectos de prácticas. Resulta, por lo tanto, un campo de estudio a seguir explorando y profundizando. En otras palabras, es necesario desarrollar otras investigaciones que posibiliten comprender qué ocurre con la formación en los sujetos que transitan un profesorado, qué aprenden en relación con la enseñanza.

\section{Método}

El objetivo general de la tesis fue identificar y describir los aprendizajes alcanzados en relación con la enseñanza por los egresados de la carrera Profesorado Universitario de Comunicación Social de la Universidad

Nacional de Córdoba durante el período 2016/2017. Esta carrera reviste el

carácter de "formación consecutiva" ya que se trata de un ciclo de "complementación curricular", destinado a graduados universitarios que aspiran a obtener un título docente. Este ciclo es posterior a la carrera de grado y consiste en una propuesta académica que integra una formación pedagógica general, una formación orientada a la enseñanza de la disciplina y prácticas en contextos de trabajo.

Interesó indagar qué y cómo reconocen haber aprendido los egresados. Responder estos interrogantes fue posible a través de un diseño metodológico de tipo cualitativo por cuanto lo que interesó estudiar no era una realidad dada sino lo que diferentes actores construyen acerca de ella, "una realidad que no existe fuera de los puntos de vista subjetivos o socialmente compartidos" (Flick, 2004, p. 41 44). Los actores elegidos para construir "la realidad" fueron los egresados y profesores de la carrera. Se propuso comprender o reconstruir la construcción (Schutz, citado por Flick, 2004) del fenómeno en cuestión -los aprendizajes reconocidos en relación con enseñanza- desde el interior, "explorándolo desde la perspectiva de los participantes en un ambiente natural y en relación con su contexto" (Hernández Sampieri, 2014, p. 358). Se intentó explorar la forma en que los sujetos perciben y experimentan el fenómeno, sus puntos de vista, interpretaciones y significados. Esta exploración exigió una reconstrucción de las

ISSN 0213-8646 | E-ISSN2530-3791 • Revista Interuniversitaria de Formación delProfesorado, 96 (35.1) (2021), 209-226 
experiencias vividas, un esfuerzo de memoria, por tanto, también el estudio asumió un carácter sincrónico. En el marco de este enfoque se llevó a cabo un abordaje fenomenológico porque interesó explorar, describir y comprender las experiencias -comunes y distintas- de las personas en relación a un mismo fenómeno o proceso, e identificar las categorías que se presentan frecuentemente y no tanto en tales experiencias.

La técnica privilegiada para reconstruir las experiencias formativas fue la entrevista semiestructurada que se aplicó a 9 egresados y a 5 profesores de la carrera durante el año 2017. En el caso de los egresados la indagación tuvo tres momentos: la narración escrita de una experiencia de aprendizaje significativa en el marco de la carrera, la entrevista propiamente dicha y la ampliación de la desgrabación-transcripción de la entrevista. Luego se incluyó un cuarto momento no contemplado al principio: los grupos focales. Esta instancia permitió volver sobre los aprendizajes reconocidos por los egresados y poner en discusión mis interpretaciones sobre ellos.

Respecto de los profesores se decidió no sólo relevar datos sobre sus propuestas de enseñanza sino además explorar sus percepciones sobre los aprendizajes de los egresados, cuestión que se pudo integrar en el análisis y permitió complejizarlo.

\section{Resultados y Discusión}

Los resultados alcanzados permiten identificar qué aprenden los egresados de la carrera, cómo y en qué condiciones. Se destaca el valor que tienen aquellos que remiten a experiencias y vivencias.

Se pudo arribar a una conceptualización de estos aprendizajes, entendidos como construcción de conocimientos -cognitivos/vivenciales- que resultan de la articulación entre situaciones/contextos, operaciones/actividades -sobre todo de orden didáctico- y condiciones relativas a la carrera y trayectorias de los estudiantes.

En relación con los aprendizajes en los que predominan los aspectos cognitivos, los egresados pudieron apropiarse de conceptualizaciones derivadas de las pedagogías críticas de la enseñanza y de los enfoques socio constructivistas del aprendizaje. Desde estos marcos reconocen rasgos y atributos de la enseñanza, entre ellos la situacionalidad, la complejidad y la variación.

Construyeron saberes prácticos, aquellos que remiten a procedimientos y a contextos de actuación profesional, vinculados a los diferentes aspectos o facetas que integra la enseñanza, esto es, la didáctica, la organizativa y la relacional (Cols, 2011), entre ellos ocupan un lugar destacado los aprendizajes que remiten a la primera faceta, especialmente los referidos a la planificación didáctica. Sobre este asunto advierten la tensión entre diseñar una propuesta de enseñanza y desarrollarla en el contexto de clase, reconocen como condición el tiempo y los estudiantes en tanto variables fundamentales a 
considerar. Asociado a la tarea de planificación reconocen haber aprendido a seleccionar y recortar contenidos de enseñanza, así como a realizar mediaciones y construir formas de representación diversas, variadas, cuyos efectos también son diversos y variados en los aprendizajes. Las tareas de mediación que exigió "colocar el conocimiento en el aula" les posibilitó reflexionar sobre el sentido formativo del conocimiento escolar. En este sentido, aprendieron a llevar a cabo "construcciones metodológicas" que resultaron de haber articulado "las lógicas de contenido a enseñar y las lógicas de los sujetos que aprenden, mediadas por los ámbitos o contextos donde tales lógicas se entrecruzan" (Edelstein, 2011, p.147).

Respecto de los aprendizajes relativos a las facetas organizativa y relacional, reconocen la incidencia de los contextos organizativos y de los vínculos que se construyen en los procesos de enseñanza. Aluden a la necesidad de reconocer a los estudiantes como sujetos de aprendizaje y de construir confianza acerca sus posibilidades de aprender. Toman conciencia que aprender a vincularse con los estudiantes es un saber que se aprende "leyendo en las personas" el texto que ellas portan, notan que esta lectura es recíproca, ellos mismos también fueron leídos

por los estudiantes. Advierten que el vínculo con los estudiantes es una construcción, un trabajo arduo no garantizado de antemano.

El aspecto sensible y vivencial del aprendizaje fue apareciendo en las relecturas de los relatos. Prestar atención a esta dimensión permitió develar algo del misterio que tiene el aprendizaje ya que hizo posible recuperar las emociones, sentimientos, y sensaciones que les produjo a los egresados transitar el profesorado. A tal fin se propusieron nombres que dieran cuenta en parte de tales experiencias: ocupar el espacio -poner el cuerpo; mirar, imaginar, abrirse a otra cosa; verse y situarse de otro modo; dar vueltas, armar y desarmar; estar y meterse con otros-implicarse; atreverse con lo propio.

Es recurrente el aprender a estar en la clase. Para los egresados significó apropiarse de ese espacio, pararse, situarse en ese lugar, ponerse de "ese otro lado", un lugar distinto", un lugar a conquistar, un "campo de fuerzas". Sintieron tensión, temor, incertidumbre, fragilidad, el momento clave es cuando tuvieron que hacerse cargo de la clase. "Dar clase", para la mayoría de los egresados, fue "shockeante" porque implicó poner el cuerpo, mostrarse, hacerse escuchar, ser observado y sometido a juicio, sintieron que fueron medidos y que pudieron medirse. Fue la experiencia más significativa porque a través de ella pudieron dar "el paso", ingresar al territorio escolar y participar con cierta intensidad de "comunidades de práctica", interactuar con sus miembros y comprender sus valores, creencias y conductas. Porque es en el seno de esta comunidad que los egresados desarrollaron la capacidad de construir e intercambiar conocimientos para desarrollar actividades valiosas y con significado. Asumieron su lugar de "periferia", de recién llegados a un lugar que no les pertenecía aún, sin embargo, un lugar que les habilitó estar y 
participar de esa comunidad llamada escuela, con sus tradiciones, códigos y rutinas. Reconocen que fue en la interacción con los "expertos", miembros reconocidos de esa comunidad, que han logrado observar, entender, significar las prácticas de esta comunidad y actuar ahí. Desde ese lugar aprendieron a tomar decisiones por su cuenta, resolver situaciones inesperadas que debieron afrontar con sus propios recursos o inventar soluciones e "improvisar", experiencias que les mostró que enseñar tiene algo de "incalculable" (Antelo, 2005). Algunos notaron que el aula puede ser un lugar de polifonía, de emergencia de voces de docentes y estudiantes.

Todos los egresados experimentaron cambios, en tanto movimientos y alteraciones que los hizo "salirse" para aprender a pensar, significar, mirar, sentir de otra manera, cambios que habilitaron tener otra apertura respecto de la enseñanza, gracias a ello imaginar y encarar prácticas pedagógicas diferentes, más "arriesgadas", con un "tinte" de innovación. No obstante, reconocieron que les costó abandonar los formatos conocidos y vividos en su biografía escolar, armar una clase que no replique los formatos habituales.

Para la mayoría significó la ruptura de estructuras, matrices, prejuicios; "pensar la educación en otras claves". Porque aprendieron a mirar "lo que pasa en el aula", a divisar cuestiones no tan visibles y palpables. Los cambios vividos por los egresados muestran que han logrado un aprendizaje en términos de lo que expone Greene (2009), deshacerse de modos habitualesusuales de ver, pensar, actuar y construir otros modos, un proceso que es posible cuando ocurre una crisis de conciencia "lo que antes era incuestionado ahora es cuestionado, lo que estaba sumergido ahora se hace visible" (p. 83).

Se dieron cuenta que la docencia requiere reconstituir condiciones que hoy no están dadas y que esta restitución es parte de la tarea de enseñar, "el oficio de profesor consiste, pues, menos en dar clases que en construir las condiciones que permiten darlas...ya no se puede enseñar tontamente... los adolescentes ya no son conquistados a priori por el profesor" (Dubet, 2006 p.176). Dieron cuenta de la influencia que se puede ejercer a través de la enseñanza, no obstante, reconocieron sus límites que lleva a renunciar a "todo ideal de eco completo, de recepción traslúcida" (Steiner, 1991, citado por Antelo, 2005 p. 174).

Vivieron la "cocina" de la planificación educativa y su desarrollo, una tarea que implica armar, desarmar, rearmar, ir adelante y hacia atrás, "dar vueltas". Tomaron conciencia que una propuesta didáctica nunca es una obra acabada y que sólo se pone a prueba en el aula porque la enseñanza se realiza "a condición de no poder saber nada a priori acerca del resultado final" (Antelo, 2005, p.174).

Tuvieron que "invertir en el trabajo su personalidad, emociones, sentimientos y pasiones, con todo lo que ello tiene de estimulante y riesgoso al mismo tiempo" (Tenti Fanfani, 2008, p.40), las experiencias relatadas hablan de esta inversión, cuánto de su personalidad, sus miedos, 
incertidumbres, ansiedades, se pusieron en juego. Algunos egresados manifestaron la sensación de fragilidad y vulnerabilidad que les produjeron sus prácticas en el aula.

Experimentaron que la enseñanza en una acción intersubjetiva que obliga a "meterse con otros". Aprendieron a trabajar con estudiantes, compañeros, profesores. Respecto de los estudiantes tuvieron que prestar atención a sus reacciones y preguntas, lo que hizo ruido, saber esperar, aceptar sus tiempos, "bancarse" lo inesperado, lo emergente, en algunos casos tener que "salirse" y "negociar".

Reconocen la incidencia de la subjetividad en las decisiones de enseñanza, muchas de ellas tienen que ver con uno mismo, con las propias historias, ideas, gustos, posicionamientos, visiones, convicciones, estilos personales, sentimientos de quienes enseñan. Los egresados notaron que existe un margen de libertad para crear formas de enseñar y construir versiones propias. Son conscientes de que han logrado elaborar propuestas singulares y producir un modo personal de intervención.

Los aprendizajes referidos dan cuenta de la dimensión tácita del saber docente, ese "saber hacer constituido por principios y pautas de actuación vinculados a contextos escolares concretos y poco vinculados con los conceptos y teorías de las ciencias de la educación" (Porlan Ariza, 1994, p. 50) De las metáforas que utilizan los egresados para hablar de sus experiencias se desprenden estos saberes: "meterse muy finito en la clase", "mirar el paisaje que se iba componiendo" "darme cuenta de la magia", "decidir sobre la marcha", "tener un termómetro", "dar vueltas", "ir y venir", "enredarse" "salirse del corset", "desmenuzar".

Es recurrente la referencia acerca de que la enseñanza es una práctica compleja, los egresados vivieron esta complejidad y los profesores reconocen la dificultad de enseñar y aprender esta práctica. Aluden a las manifestaciones de la complejidad: incertidumbre e imprevisibilidad; singularidad, alteridad, contingencia. Notan la mutación/reconfiguración de los problemas de enseñanza lo que demanda invención y adaptación según las situaciones, una intervención social con producción de efectos, un acto responsable. Toman conciencia que la enseñanza involucra emociones que definen y expresan significaciones y horizontes, un tipo de mediación que implica algún tipo de conexión no garantizado de entrada entre estudiantes y el conocimiento. En relación a ello, advierten que aprender a enseñar no es un asunto que se resuelve sólo "técnicamente", especialmente plantea la exigencia de interpelar posicionamientos políticos y éticos sobre todo en las instancias de intervención profesional, aquellas que les mostraron que "las cosas pasan de otra manera".

Respecto de las situaciones y contextos se observa la importancia de ingresar y estar en el aula e integrarse a un grupo/comunidad, cobra importancia la situacionalidad y la colaboración/cooperación. La valoración 
del contexto y de lo grupal refuerza la concepción de aprendizaje sostenida desde las perspectivas socio cognitivas y contextualistas (Rogoff, 2012, Baquero, 2012)

Todos los egresados consideran que las prácticas en las instituciones fue la instancia más significativa de toda la carrera, el momento en el que lograron los aprendizajes que buscaban lograr, aquellos vinculados específicamente al desempeño de un profesor.

Las prácticas se realizaron principalmente en el sistema educativo formal. En estos contextos, comprendieron de qué se trata enseñar, la complejidad de esta acción; no se trata ya de escucharlo, leerlo o decirlo.

Advierten la diferencia entre observar, leer, discutir sobre los contextos escolares y de aula y estar en esos contextos, reconocen que sólo es posible comprender esta tarea cuando se está en el aula, es un aprendizaje situado que se construye en contexto y a través de la experiencia. Las actividades de prácticas son importantes porque en ellas han podido resignificar algunos planteos teóricos, en esas situaciones "les cayó la ficha", las reconocen como instancias en las que se juega la formación "en serio", ahí se "miden", se dan cuenta si podrán ser docentes. La resistencia que reconocen haber tenido ante tanta exigencia de parte de los profesores aminora cuando alcanzan a comprender el sentido de lo que se les demanda.

Valoran la riqueza del trabajo colaborativo, haber constituido equipos de trabajo integrados por practicantes, profesor orientador y profesor de prácticas, sintieron que fue una experiencia de la que se "se salió enriquecido". Desarrollaron la capacidad de hacer converger puntos de vista, implicarse en la tarea, afrontar con otros la enseñanza en los actuales escenarios. Aprendieron maneras de hacer docencia, a operar en contextos complicados, hubo un aprovechamiento de la experiencia-aprendizaje del profesor "experto" y también de los compañeros practicantes. Asimismo, advierten la complejidad de lo grupal, la convergencia es una tarea ardua, desata encuentros y desencuentros, órdenes y desórdenes, puntos de unión y separación.

Sobre las operaciones y actividades se destacan aquellas que promueven la producción y reflexión didácticas. Estas operaciones fueron: la reconstrucción de conocimientos sobre la formación de base; la reflexión sobre los conocimientos de base en clave didáctica; la reflexión sobre las propias prácticas; la observación de prácticas y contextos; la resolución de problemas de las prácticas y toma de decisiones; la reelaboración de propuestas de enseñanza.

Se observa el intento por articular los conocimientos de base con los didácticos en orden a construir conocimiento pedagógico, es decir, "transformar el conocimiento de la materia en formas que sean didácticamente impactantes y aun así adaptables a la variedad que presentan los alumnos en cuanto a habilidades y bagajes" (Shulman, 2005, p.21). A fin de 
alcanzar dicha articulación llevaron a cabo la lectura de prescripciones curriculares, la puesta en diálogo entre sus marcos teóricos y dichas prescripciones, la selección de perspectivas teóricas respecto de las temáticas a enseñar, la selección de conceptos y su mapeo para reflejar posibles conexiones ente ellos, la definición -formulación de ejes temáticos, el recorte/delimitación de temas y su vinculación con el contexto de los estudiantes y de la escuela, la elaboración de materiales y guías a modo de mediación, la selección o producción de recursos que faciliten la comprensión de los estudiantes, la tarea de "trasladar la complejidad de la temática a actividades simples".

La observación de prácticas y contextos forma parte del proyecto de prácticas profesionalizantes, reconocen que gracias a esta tarea fue posible llevar a cabo el trabajo didáctico. Sienten que al principio "estaban perdidos", que "fueron llevados" a mirar sin rumbo, a prestar atención a cosas que tenían que ver con ellos mismos, con sus historias, sus sentimientos y emociones. En el transcurso aprendieron a focalizar en los aspectos significativos para sus intervenciones inmediatas.

Se advierte a su vez el empeño por promover procesos cognitivos consonantes con los modelos de formación centrados en el análisis y reflexión (Ferry, 2008; Schön, 1992; Edelstein, 2011). Mencionan diferentes "objetos de análisis": la institución escuela y los contenidos, concepciones y perspectivas, los aprendizajes en las prácticas, las condiciones de aprendizaje en los contextos escolares. Poner en análisis estas cuestiones probablemente permitió a los egresados realizar rupturas en las maneras de concebir y hacer la enseñanza, aspecto señalado en párrafos anteriores. Es de destacar el trabajo de autoanálisis que pudieron realizar en línea con el planteo de Filloux (2005) un trabajo inter e intrasubjetivo. A partir de ello, fue posible analizar sus propios aprendizajes, cuestión que notan es necesario trabajar en la docencia, en este sentido, reconocen que no se puede enseñar si uno no reflexiona sobre sus saberes.

La mayoría de las actividades requirieron de la escritura, se asombran de que la docencia exija escribir tanto. Aprendieron a escribir planificaciones, documentar y narrar prácticas, es decir, se apropiaron de los géneros propios de la escritura profesional de los docentes.

En relación con las condiciones, operó como facilitador de los aprendizajes las propuestas de cátedra y las trayectorias formativas y profesionales de los estudiantes.

Respecto de las propuestas de cátedra, se valoran aquellas que promueven un trabajo de vinculación con la práctica de referencia (Martinand, 1994).

Dichas propuestas procuran vincular teorías con los saberes prácticos, se asume que las primeras deberían operar como marco referencial para la intervención didáctica. Sin embargo, en algunos casos las categorías que 
aporta al marco teórico son aprovechadas más por su potencial explicativo que como herramientas para las decisiones de las prácticas, debido en parte a que su abordaje resulta muy general y de cierta abstracción.

Se nota un intento de promover reflexión epistemológica y didáctica orientada a problematizar el sentido social de los saberes y en espacial de los contenidos escolares, sin embargo, no parece que ella sea resultado de un trabajo colaborativo de los distintos espacios curriculares de la carrera. Estas actividades de reflexión en general son realizadas en el taller de práctica cuando los estudiantes tienen que resolver la clase, es un trabajo atravesado por la urgencia que imprime las prácticas profesionalizantes en los profesorados. Profesores y egresados señalan que este abordaje debiera ser realizado previamente o en simultáneo con las instancias de prácticas y no sólo en las prácticas. Los profesores reconocen que es necesario incorporar, sistematizar y formalizar esta tarea y transformarla en contenido transversal. Se señala que la articulación entre saberes didácticos y del campo de la comunicación es posible en la medida que las cátedras integren profesores con ambos perfiles -comunicadores y pedagogos.

El tratamiento de los contenidos se realiza desde perspectivas socio críticas y constructivistas, se enfatiza la dimensión ético y política de la enseñanza. Si bien se reconoce la dificultad para traducir la visión o propósitos políticos y éticos en prácticas docentes concretas en las aulas los ejemplos de prácticas que ofrecen los egresados muestran apuestas interesantes de traducción de tales propósitos, podría decirse que esta perspectiva no sólo es una clave de lectura y análisis de prácticas también es un marco para la producción de propuestas de enseñanza, su desarrollo y las formas de vinculación en el aula.

La modalidad de clase tradicional no es el modo preponderante de los espacios curriculares que participaron del estudio. En ellos, según narran los profesores a cargo y los egresados, las formas de trabajo se acercan más a metodologías de corte inductivo, estudio de casos, trabajo de campo, producción de proyectos, taller, simulaciones.

Las actividades que realizan en las prácticas revelan una serie de actividades formativas dentro de un encuadre de trabajo explícito que liga a los practicantes con el oficio de enseñar.

Los profesores-formadores exponen las dificultades que desafían su tarea. Remiten a la tensión entre corregir/evaluar y acompañar un

practicante como dos acciones que parecen contraponerse. En este sentido, se plantea el significado de acompañar y sus límites en el marco de una carrera de formación inicial. Para algunos profesores acompañar supone la construcción de un vínculo pedagógico que sostenga a los practicantes en función de lo que requiere, necesite cada uno. Para otros implica "un trabajo de conversación" (Camilloni, 2009) a partir de preguntas, inquietudes, dudas que se plantean en torno a la enseñanza. 
Las trayectorias formativas y profesionales de los estudiantes también son una condición ya que incide en los aprendizajes alcanzados dando lugar a diferencias significativas entre los mismos según el tipo de recorrido académico realizado en la carrera previa y sus experiencias profesionales. La formación disciplinar de base, que según el planteo de algunos profesores es débil, es un asunto que los interpela fuertemente en la medida que pondría en riesgo su trabajo como formadores ya que advierten que no todos alcanzan los aprendizajes esperados. El hecho de que algunos estudiantes del profesorado hayan realizado una especialidad durante su carrera de grado más cercana al campo educativo o que estén trabajando como docentes coloca a estos sujetos en "ventaja" respecto a quienes no cuentan con estos recorridos. Esta situación ha provocado, de acuerdo con lo que relatan los profesores, la inclusión en algunas cátedras de actividades que acompañan la diversidad de trayectorias.

Uno de los hallazgos considerados más relevante es haber arribado a una conceptualización focalizada en la particularidad de los aprendizajes alcanzados en la carrera de formación de profesores de carácter consecutivo o de complementación curricular. Se propuso la categoría "aprendizajepasaje/s" porque describe/explica las experiencias formativas de quienes transitan estas carreras. Un aspecto predominante es el pasar de un estado a otro, principalmente de comunicador a profesor y traspasar fronteras -de la universidad al mundo escolar lo cual exigió reconfigurar-se en su identidad profesional.

Ayudó entender la particularidad de estos aprendizajes la idea de rito de paso, concepto central de la teoría desarrollada por Arnold Van Gennep (2008) que supone un estado liminal, es decir, una situación incierta y ambigua.

De los ritos identificados por este autor se consideraron dos: el paso material y el rito de iniciación.

El paso material implica el paso de un territorio a otro, de un espacio a otro, cabalgar entre dos mundos. Resulta sugerente para comprender la experiencia vivida por los que estudiaron la carrera, tener que introducirse en otro mundo, el mundo de las escuelas, allí juegan a ser

docentes, aunque siguen siendo estudiantes en la universidad. Situación que informa acerca de la ambigüedad de su lugar, un estado liminal. Pasar de estudiante a docente importa complejidad ya que se les exige que, en un tiempo muy acotado, piensen y actúen como profesores cuando hasta hace poco y en simultáneo son alumnos.

En su pasaje de comunicador a educador tuvieron que reconfigurar su objeto de trabajo, respecto de la enseñanza, ensayar otras maneras de relación con el conocimiento, ya no se trata de "saber para rendir examen" o "de saber para mi propio regocijo" sino "saber para transmitir a otros" (Bombini, 2012, p.5). Se reconoce que este cambio de sentido en la relación con el saber 
constituye una alteración que interpela a los estudiantes de profesorados (Terigi, 2009; Bombini, 2012; Salgueiro, 2016).

El paso de comunicador a profesor no implicó como plantea Esteve (2006) la construcción de una identidad docente falsa, los egresados no se ven a sí mismos principalmente como un experto disciplinar y subsidiariamente como alguien que enseña. La mayoría de los entrevistados consideran que su saber de base es una herramienta que les permitió fortalecerse en el rol de profesor, dan cuenta de la existencia de cierta sinergia, cooperación entre un campo y otro, podría decirse que los egresados cabalgan entre ambos roles, esta situación les permitió construirse como comunicadores-educadores, integrarse a partir de esta doble pertenencia.

Dar clase fue el momento más significativo, una experiencia que forma parte de la memoria iniciática de los profesores (Bombini, 2012) en este sentido, propongo pensar esta experiencia como rito de iniciación. La clase es una ceremonia que los adentró a "un nuevo conjunto de símbolos, a una renovada concepción de la existencia y de participación en el mundo" (Jáuregui, 2002, p.63). Transitar la clase para muchos de ellos fue la "prueba de fuego", la instancia que les permitió iniciarse y construirse como docentes. No obstante, reconocen que es una práctica protegida, cuidada, observada, controlada, acompañada; para algunos, es una ficción, porque sienten que están de paso o en tránsito, la clase es un lugar prestado, no les pertenece, allí las reglas de juego las ponen otros, son extranjeros. El cambio de estado se daría en el ingreso al territorio laboral, en el que se traspasa la frontera, este es un paso que los transformarían en "profesores de verdad".

Aprendizaje- pasaje/s implica en casi todos los casos la presencia de "otros", profesores-tutores de cátedra, profesores orientadores, compañeros y estudiantes. Se logra una "comunión" aunque sea por un tiempo limitado, los lazos que los unen son fuertes, constituyen equipos de trabajo, en su seno todo es compartido.

Los tutores son los portavoces y mensajeros, los inician en el contacto con la comunidad escolar, preparan el terreno para su paso.

Acompañan desde una confianza habilitante de la escucha y del decir, del preguntar, del comprender y al mismo tiempo del saber poner límites.

Los profesores orientadores son los anfitriones y representantes de la comunidad, autorizados para recibir y acompañar. Tienen el poder de escoger, recibir, filtrar, seleccionar a los visitantes, otorgar el derecho de visita, de ser huésped. La mayoría muestra hospitalidad, aminorando la delimitación de los umbrales y fronteras entre lo extranjero y lo no extranjero (Derrida, 2008). Se ocupan de dar a conocer las reglas y normas y hacerlas cumplir. También hacen donaciones y regalos, su saber y el fruto de su trabajo.

Cada practicante contó con compañeros de viaje, sus pares, fue una experiencia educativa de alteridad, que supuso conectarse con un otro distinto, aprender en las diferencias y desde la diferencia construir un 
nosotros.

Tuvieron que sortear obstáculos y barreras, entre ellos el comportamiento de los estudiantes cuando desafían los códigos y normas de la comunidad escuela y a sus representantes. Los estudiantes de los espacios de prácticas los "ponen" en ese lugar, ellos los llaman "profes" y los reconocen como tales.

\section{Conclusiones}

Los resultados refuerzan lo que la literatura especializada expone acerca del aprendizaje en relación con la enseñanza en contextos de formación, por un lado, el potencial formativo que tiene los espacios de prácticas de ahí la necesidad de vincular las propuestas de formación con las prácticas de referencia (Martinand, 1994) y los contextos de desempeño profesional, por otro, la fuerza del trabajo colaborativo que implica consensuar y hacer converger puntos de vistas, saberes y experiencia.

Los egresados valoran los enfoques situacionales y contextualistas centrados en la práctica cooperativa (Rogoff, 2012) y reflexiva (Schön, 1992), una formación desde dentro de la profesión (Nóvoa, 2009). Reconocen la necesidad de pasar del ámbito universitario al escolar, apropiarse de ese espacio, "pararse y estar en la clase" y participar con cierta intensidad de "comunidades de práctica". En estos contextos fue posible efectuar el trabajo didáctico que solicita la docencia, como expresan los egresados, "colocar el conocimiento en el aula".

Estas conclusiones animan a reflexionar en torno a los aspectos didácticos de la formación de profesores, es decir, la enseñanza que desarrollan las carreras de profesorados orientada a la construcción de aprendizajes en relación con la enseñanza. A replantear, entre otras

cuestiones, cuánto de lo que se propone en los programas de cátedras incluye y da cuenta de lo que para los egresados resulta significativo, si las maneras de construcción de conocimiento que se proponen en estas carreras son propias, adecuadas y pertinentes para la construcción de los saberes que demandan la práctica, que se sabe no

son las mismas que las del conocimiento académico y formalizado.

Asimismo, problematizar ¿qué aprovechamiento se hace de las prácticas observadas en los contextos de prácticas? ¿qué dispositivos

de análisis se proponen? En otras palabras, ¿qué abordaje se realiza de los componentes de una práctica profesional como es la docencia?

La formación inicial de profesores sigue siendo objeto de debate en los ámbitos académicos y políticos, no obstante, es una problemática no del todo resuelta en las propuestas de enseñanza y en los proyectos de prácticas. Resulta, por lo tanto, un campo de estudio e intervención a seguir explorando y profundizando, particularmente, los aprendizajes efectivamente alcanzados. Los estudios que se aproximan a esta temática lo hacen desde una perspectiva 
más bien normativa que intenta informar sobre las expectativas acerca de la formación recibida, sobre lo que se espera o debiera aprender en relación con los saberes que se supone debe adquirir un sujeto que se prepara para el ejercicio docente. La indagación acerca de lo que se reconoce efectivamente aprender, "lo que es y no tanto lo que debiera ser".

Se destacan los aspectos sensibles/vivenciales del aprendizaje: poner el cuerpo, mirar, verse y situarse de otro modo, asumir lo propio. Dichos aspectos tan valorados por los participantes del estudio ofrecen una vía de indagación interesante, no sólo porque posibilita aproximarse a la comprensión y sistematización de tales aprendizajes, no del todo explícitos en un proceso formativo, además porque permite problematizar el lugar que ocupan dichos aprendizajes en las propuestas de cátedra, la consideración de estos aspectos en la enseñanza en los espacios de formación. En esta línea es posible construir conocimiento acerca de los dispositivos que promueven experiencias formativas significativas en relación a aprender a enseñar y a enseñar a enseñar identificando los elementos, rasgos, condiciones que las favorecen, insumo relevante para reorientar el curriculum de formación y la formación de los formadores.

Ciertamente la investigación es una actividad inagotable, infinita, porque sus resultados permiten construir nuevos puentes y zonas de indagación. El estudio sobre los aprendizajes, la enseñanza, la formación docente vuelve a plantearse como una necesidad de seguir siendo explorada.

\section{Referencias}

Antelo, E. (2005). Notas sobre la (incalculable) experiencia de educar. En Frigerio, G. y Diker, G. (Ed.), Educar: ese acto político. (pp. 173-183). Buenos Aires, Argentina: Del Estante Editorial.

Baquero, R. (2012). Alcances y límites de la mirada psicoeducativa sobre el aprendizaje escolar: algunos giros y perspectivas. Polifonías Revista de Educación. 1(1), 9-21.

Bombini, G. (2012). Escribir la metamorfosis. Escritura y formación docente. Buenos Aires, Argentina: Ediciones El Hacedor.

Camilloni, A. (2009). Prólogo. En Anijovich, R. et. al. (Ed.) Transitar la formación pedagógica: dispositivos y estrategias (pp.13-20). Buenos Aires, Argentina: Paidós.

Cisternas. T. (2011). La investigación sobre formación docente en Chile. Territorios explorados e inexplorados. Revista Calidad en la Educación, 35, 131-164.

Cols, E. (2011). Estilos de enseñanza. Rosario, Argentina: Homo Sapiens.

Davini, C. (2015). La formación en la práctica docente. Buenos Aires, Argentina: Paidós.

Derrida, J (2008). La Hospitalidad. Buenos Aires, Argentina: Ediciones de la Flor.

Dubet, F. (2006). El declive de la institución. Profesiones, sujetos e individuos en la 
modernidad. Barcelona, España: Gedisa

Edelstein, G. (2011). Formar y formarse en la enseñanza. Buenos Aires, Argentina: Paidós.

Esteve J. M (2009). La profesión docente ante los desafíos de la sociedad del conocimiento En Vélaz de Medrano, C- y Vaillant, D. (Coords.) Aprendizaje y desarrollo profesional docente (pp.17-28) Madrid, España: Fundación Santillana, OEI.

Ferry, G. (2008). Pedagogía de la Formación. Buenos Aires, Argentina: Ediciones Novedades Educativas.

Filloux, J. C. (2005). Intersubjetividad y formación. Buenos Aires, Argentina: Ediciones Novedades Educativas.

Flick, U. (2004). Introducción a la Investigación Cualitativa. Madrid, España: Morata.

Greene, M. (2009). El profesor como extranjero. En Larrosa, J. et al. Déjame que te cuente (pp. 81-130). Barcelona, España: Laertes.

Hernández Sampieri, R. (2014). Metodología de la investigación. México, México: Mc. Graw Hill Education.

Jáuregui, J. (2002). La teoría de los ritos de paso en la actualidad. Antropología. Revista Interdisciplinaria Del INAH, 68, 61-95. https://www.revistas.inah.gob.mx/index.php/antropologia/article/view/4 $\underline{971}$

Martinand, J.L. (1994). La didáctica de las ciencias y la tecnología y la formación de profesores. Revista Investigación en la escuela, 24, 59-69.

Nóvoa, A. (2009). Para una formación de profesores construida dentro de la profesión. Revista de Educación, 50, 203-218.

Porlan Ariza Rafael (1994). El saber práctico de los profesores especialistas. Aportaciones desde las didácticas específicas. Revista Investigación en la escuela, 24, 49-58.

Rogoff, B. (2012). Learning without lessons: Opportunities to expand knowledge. Journal for the Study of Education and Development, 35(2), 233-252, http://dx.doi.org/10.1174/021037012800217970

Salgueiro, A. (2016). ¿Qué se aprende sobre la enseñanza en la carrera de formación de profesores? El caso profesorado en Comunicación Social de la Universidad Nacional de Córdoba, Argentina. Espiral, Revista de Docencia e Investigación, 6(1), 137 - 148.

Schön, D. (1992). La formación de profesionales reflexivos. Hacia un nuevo diseño de la enseñanza y el aprendizaje de las profesiones. Barcelona, España: Paidós.

Shulman, L. (2005). Conocimiento y enseñanza: fundamentos de la nueva reforma. Revista de currículum y formación del profesorado, 9, 2 pp 1-30. 
http://www.ugr.es/local/recfpro/Rev92ART1.pdf.

Tenti Fanfani, E. (2008). Reflexiones sobre la construcción social del oficio docente. En Vélaz de Medrano. C. y Vaillant, D. (Ed.) Aprendizaje y desarrollo profesional docente (pp. 39-48). Madrid, España: Fundación Santillana, OEI.

Terigi, F. (2009). La formación inicial de profesores de Educación Secundaria: necesidades de mejora, reconocimiento de sus límites. Revista de Educación, 350 ,

123-

144.http://www.revistaeducacion.educacion.es/re350/re350_06.pdf

Van Gennep, A. (2008). Los ritos de paso. Madrid, España: Alianza Editoria 
